\title{
Magnetic resonance imaging assessment of cerebral small vessel disease intensification in patients with severe aortic valve stenosis
}

\author{
Jakub Chrostowski ${ }^{1 A, B, C, D, E, F, G}$, Marcin Majos ${ }^{2 C, D, E, F}$, Andrzej Walczak ${ }^{3 B, D, G}$, Mariusz Wachowski ${ }^{1 C, D, F}$, Agata Majos ${ }^{1 A, B, D, F, G}$ \\ 'Department of Radiological and Isotopic Diagnosis and Therapy, Medical University of Lodz, Poland \\ ${ }^{2}$ Department of Radiology and Diagnostic Imaging, Medical University of Lodz, Poland \\ ${ }^{3}$ Department of Cardiac Surgery, Medical University of Lodz, Poland
}

\section{Abstract}

Purpose: To assess the prevalence of the neuroradiological indices of cerebral small vessel disease (CSVD) in patients with severe aortic valve stenosis (AS) in magnetic resonance imaging (MRI).

Material and methods: 34 patients (age 60-90 years, 17 women and 17 men) with severe AS and 50 healthy controls (age 61-85 years, 29 women and 21 men) underwent MRI brain examinations, which were analysed for the neuroradiological indices of CSVD: hyperintensities in periventricular white matter (PVWM) and deep white matter (DWM), enlarged perivascular spaces (ePVS), lacunar strokes, and cerebral microbleeds (CMBs).

Results: PVWM hyperintensities were found in $46 \%$ of volunteers and was significantly lower $(p=0.027)$, corresponding to AS patients ( $80 \%)$, the density of lesions was higher in the AS group than in controls $(p=0.019)$. DWM hyperintensities were found more often in AS patients (76\%) than in controls $(66 \%)(p=0.303)$, but the densities were similar in both groups. Lacunar strokes were found in $35 \%$ of AS patients and $16 \%$ of controls $(p=0.042)$. The average number of lacunar strokes per person was 0.9 in the AS group and 0.3 in the controls $(p=0.035)$. The AS group showed higher variance in the number of strokes: $\mathrm{SD}=1.96 \mathrm{vs.} \mathrm{SD}=1.06$ in controls. Both prevalence and density of the ePVS and CMBs did not differ significantly between the groups.

Conclusions: Neuroradiological indices of the vascular disease do not provide an unequivocal clue to the pathogenesis of CSVD in patients with severe AS. Most observations imply that CSVD is primarily a consequence of cerebral hypoperfusion caused by AS.

Key words: aortic stenosis, cerebral small vessel disease, lacunar stroke, perivascular spaces, cerebral microbleed, white matter hyperintensities.

\section{Introduction}

Because of the growing popularity of preventive healthcare and a healthy lifestyle, as well as considerable progress in the diagnosis and treatment of cardiovascular diseases, the contribution of the latter to overall mortality has substantially decreased over the last 3 decades. Nevertheless, cardiovascular diseases still constitute the leading cause of mortality in Poland, with conditions from this group contributing to nearly every second death [1]. The most common valvular disease among cardiovascular conditions is aortic valve stenosis (AS). The incidence of AS is associated with age and shows a steady growing tendency [2]. The causes of AS mainly comprise calcific stenosis of a tricuspid valve, a bicuspid aortic valve with imposed calcific changes, and rheumatic valve disease. Doppler echocardiography is the key tool for the diagnosis and evaluation of AS and is the primary non-invasive imaging method for

Correspondence address:

Dr. Jakub Chrostowski, Department of Radiological and Isotopic Diagnosis and Therapy, Medical University of Lodz, 251 Pomorska St., $92-213$ Lodz, Poland,

e-mail: jchrostowski90@gmail.com

Authors' contribution:

A Study design · B Data collection · C Statistical analysis · D Data interpretation · E Manuscript preparation · F Literature search · G Funds collection 
AS assessment. The severity of valve stenosis can be graded in echocardiography as mild, moderate, or severe and depends on aortic peak jet velocity, mean aortic valve gradient (AVG), and aortic valve area (AVA). Peak jet velocity $\geq 4 \mathrm{~m} / \mathrm{s}$, mean AVG $\geq 40 \mathrm{mmHg}$, or AVA $<1.0 \mathrm{~cm}^{2}$ are consistent with severe aortic stenosis [3].

Chronic AS hinders outflow of the blood from the left ventricle, and causes an increase in left ventricular pressure and left ventricular hypertrophy as a compensatory mechanism to maintain normal stroke volume. Another protective mechanism is the constriction of peripheral vessels in non-vital tissues and organs to enhance blood flow in other vessels, among them cerebral arteries [4]. Despite the activation of the compensatory mechanisms, the brain may be exposed to chronic hypoperfusion, which leads to structural changes in small blood vessels supplying the white matter and deep structures of the grey matter. Being predominant among several various processes, this mechanism is postulated to constitute the basis for the development of cerebral small vessel disease (CSVD), an entity involving small arteries, arterioles, capillaries, and venules [5]. CSVD causes characteristic neurological manifestations and specific changes visible on neuropathological examination and neuroimaging. The specific radiological features of CSVD include white matter hyperintensities, enlargement of perivascular spaces, lacunar strokes, microbleeds, and cerebral atrophy [6].

Previous studies demonstrated the influence of cardiovascular diseases, such as arterial hypertension, atherosclerosis of thoracic aorta, internal carotid arteries and branches of large perforating arteries of the brain (branch atheromatous disease - BAD), transient ischaemic attacks (TIAs), and previous stroke on various components of CSVD [7-9]. It is still unclear whether the most common valvular disease, AS, also plays a role in CSVD pathogenesis. From both a clinical and social perspective, it is essential to verify whether chronic AS plays a role in the development of CSVD. Thus, the aim of this study was to analyse the prevalence of the neuroradiological indices of CSVD in patients with severe AS.

\section{Material and methods}

This prospective study was approved by the Local Bioethics Committee, and informed consent was obtained from each participant prior to the examination. The study included 34 patients with severe AS qualified for transcatheter aortic valve implantation (TAVI) at the Department of Cardiosurgery, Medical University of Lodz (Poland). This group consisted of 17 women and 17 men, with a mean age of 76 years (range: 60-90 years). Additionally, a control group of 50 volunteers ( 29 women and 21 men) with a mean age of 73 years (range: 61-85 years) was included. The control group consisted of healthy volunteers who underwent screening tests for cardiovascular pathology in our hospital within last 2 years.
Patients with severe AS presented with relatively good, good, or very good clinical status. Thirty patients reported at least one symptom typical of advanced AS, most often exertional dyspnoea $(n=30)$, followed by general weakness $(n=23)$, heart palpitations $(n=12)$, dizziness $(n=11)$, dyspnoea at rest $(n=9)$ and/or dry cough $(n=4)$. Four patients with severe AS reported no symptoms typical of this condition. All controls presented with very good clinical status, with no symptoms typical of AS. All participants of the study had chest X-ray. Patients with severe AS had an echocardiography and Doppler ultrasound of carotid and vertebral arteries during hospitalization. Each volunteer of the control group had medical record documentation, including echocardiography and Doppler ultrasound of carotid and vertebral arteries obtained from screening tests.

Inclusion criteria for the AS group included severe AS confirmed in echocardiography, and for the control group: absence or mild AS confirmed in echocardiography within the last 2 years. Exclusion criteria for both groups included, confirmed within last 2 years, concomitant significant cardiac pathology other than AS in echocardiography, significant defect of the carotid or vertebral artery in Doppler ultrasound, also cerebral pathology limiting MR scans assessment, e.g. past ischaemic stroke.

Each participant of the study underwent MRI of the brain at the Department of Procedural Radiology and Ultrasonography, Central Clinical Hospital, Medical University of Lodz. The studies were carried out with a Siemens Magnetom Avanto 1.5T MRI scanner using the following sequences: 3D T1 (TE: $3.4 \mathrm{~ms}$, TR: $2200 \mathrm{~ms}$, FOV read: $256 \mathrm{~mm}$, layer thickness: $1 \mathrm{~mm}$, Flip angle: $15^{\circ}$ ), T2 (TE: $100 \mathrm{~ms}$, TR: $6270 \mathrm{~ms}$, FOV read: $256 \mathrm{~mm}$, layer thickness: $1 \mathrm{~mm}$, Flip angle: $15^{\circ}$ ), FLAIR (TE: $352 \mathrm{~ms}$, TR: $6000 \mathrm{~ms}$, FOV read: $241 \mathrm{~mm}$, layer thickness: $0.9 \mathrm{~mm}$, Flip angle: $15^{\circ}$ ), SWI (TE: $40 \mathrm{~ms}$, TR: $49 \mathrm{~ms}$, FOV read: $230 \mathrm{~mm}$, layer thickness: $2 \mathrm{~mm}$, Flip angle: $15^{\circ}$ ), and DWI + ADC (TE: $91 \mathrm{~ms}$, TR: 3500 ms, FOV read: $230 \mathrm{~mm}$, layer thickness: $5 \mathrm{~mm}$ ).

Image analysis was performed by 2 radiologists: a radiologist in training and an expert in neuroradiology with 15 years' experience, who were blinded to the participants. In our study the standard approach in reporting neuroimaging findings proposed in CSVD based on the Standards for Reporting Vascular changes in neuroimaging (STRIVE) was used [6]. The MRI scans were analysed for the following neuroradiological indices of CSVD:

- white matter hyperintensities (WMH) in the periventricular white matter (PVWM) and deep white matter (DWM) (Figure 1); the size and confluence of the lesions were graded using the scale proposed by Fazekas et al. [10];

- lacunar strokes (Figure 2); the analysis included all lacunar strokes visible on the MRI scans, regardless of location;

- enlarged perivascular spaces (ePVS) (Figure 3); the analysis included all ePVS visible on the MRI scans. The lesions were quantified in 3 areas, semi-oval centre, basal nuclei, and mesencephalon, using the scale proposed by Maclullich et al. [11]; 
- cerebral microbleeds (CMBs) (Figure 4); presence and quantity of the CMBs were determined in 3 areas: deep cerebral structures, cerebral lobes, and infratentorial structures; the density of the CMBs was graded using the Microbleed Anatomical Rating Scale (MARS) proposed by Gregoire et al. [12].

Categorical variables are presented as the number of patients, absolute numbers, and percentages. Numerical variables were depicted by mean and standard deviation values. Statistical significance of differences in the numerical traits between the study groups was appraised by using ANOVA (for normally distributed variables) or Mann-Whitney test (for non-normally distributed ones). $P$ values less than 0.05 were considered statistically significant. All the statistical procedures were performed using Stata/Standard Edition, release 14.2 (StataCorp LLC, College Station, Texas, USA).

\section{Results}

\section{White matter hyperintensities}

PVWM hyperintensities in patients corresponded to nearly $80 \%$ prevalence. The prevalence of the PVWM hyperintensities in the controls was significantly lower $(p=0.027)$ - these lesions were found in $46 \%$ of volunteers. Among participants who presented with PVWM hyperintensities, the density of these lesions graded with the Fazekas scale was significantly higher in the AS group than in the controls $(p=0.019)$. Although DWM hyperintensities were found more often in patients with severe AS than in the controls, the between-group difference was not statistically significant ( $p=0.303$ ). The density of the DWM hyperintensities according to the Fazekas scale was similar in both groups, with slightly, albeit not significantly, higher prevalence of more advanced lesions in patients with severe AS. The DWM hyperintensities corresponding to Fazekas grade 3 were found solely in patients with severe AS. The results are presented in Table 1.

\section{Lacunar strokes}

The prevalence of the lacunar strokes in the AS group was more than twice as high as in the controls (35\% vs. 16\%), and the difference was statistically significant $(p=0.042)$. The average number of lacunar strokes per person was 3 times as high as in the controls $(p=0.035)$; moreover,
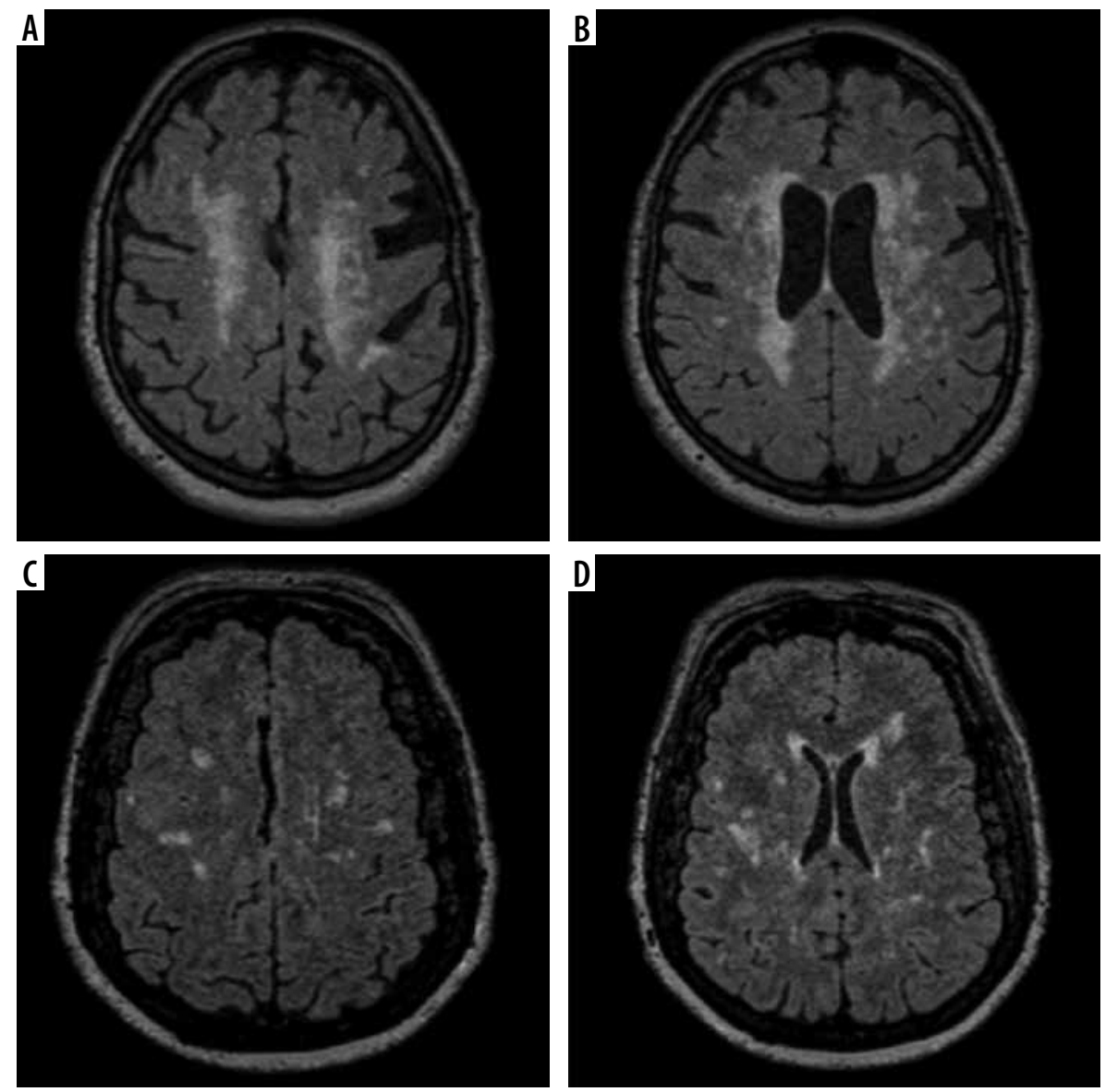

Figure 1. White matter hyperintensities. A) Transverse FLAIR image of a white matter hyperintensities (WMH) in the semi-oval centre, grade 3 according to the Fazekas scale. B) Transverse FLAIR image of a WMH in the periventricular white matter surrounding the lateral ventricles, grade 3 according to the Fazekas scale. () Transverse FLAIR image of a WMH in the semi-oval centre, grade 2 according to the Fazekas scale. D) Transverse FLAIR image of a WMH in the periventricular white matter surrounding the lateral ventricles, grade 2 according to the Fazekas scale 

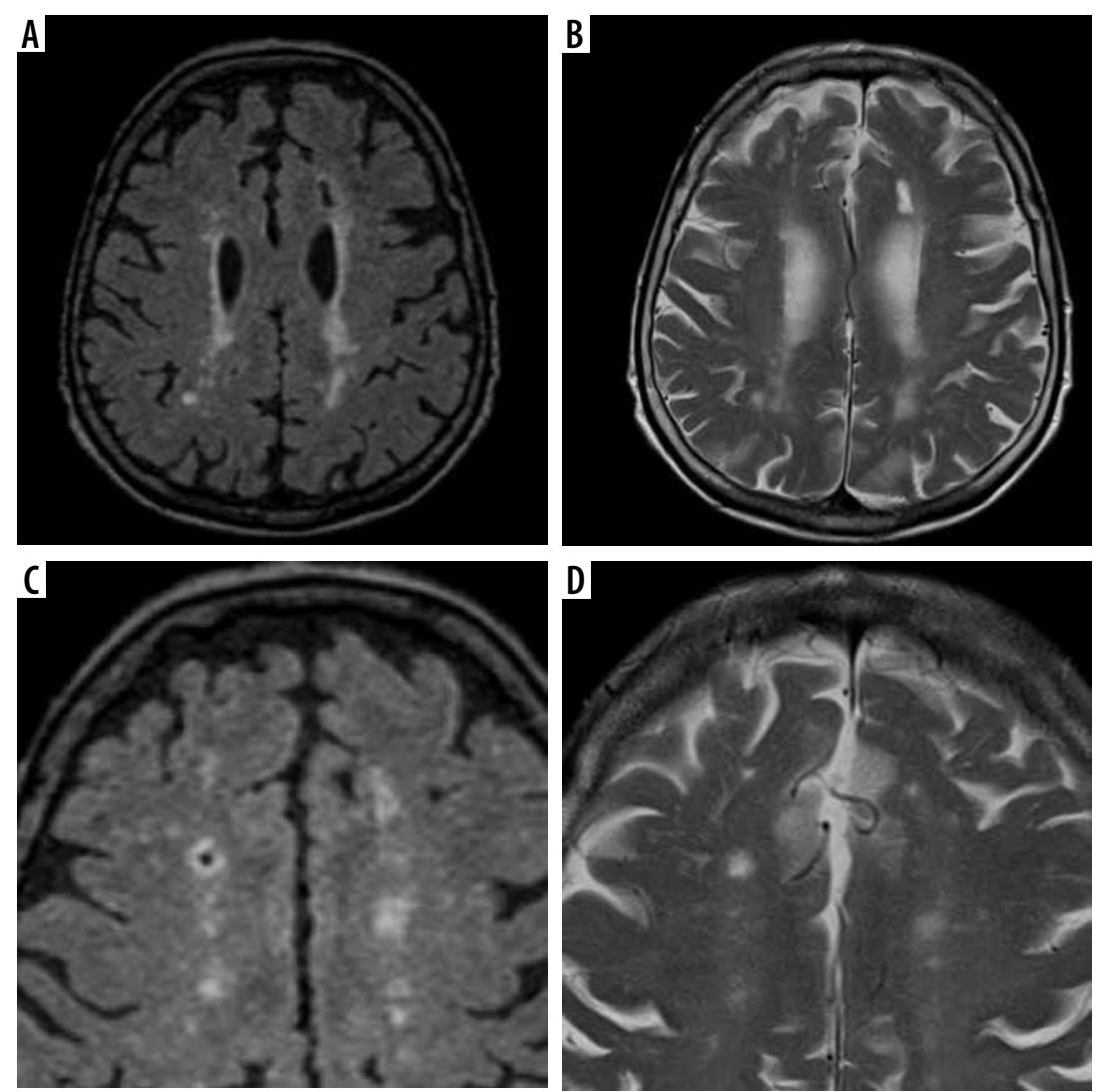

Figure 2. Lacunar strokes. A) Transverse FLAIR image of a lacunar stroke in the white matter of the left cerebral hemisphere. B) Transverse T2-weighted image of a lacunar stroke in the white matter of the left cerebral hemisphere. C) Transverse FLAIR image of a lacunar stroke in the white matter of the right cerebral hemisphere. D) Transverse T2-weighted image of a lacunar stroke in the white matter of the right cerebral hemisphere
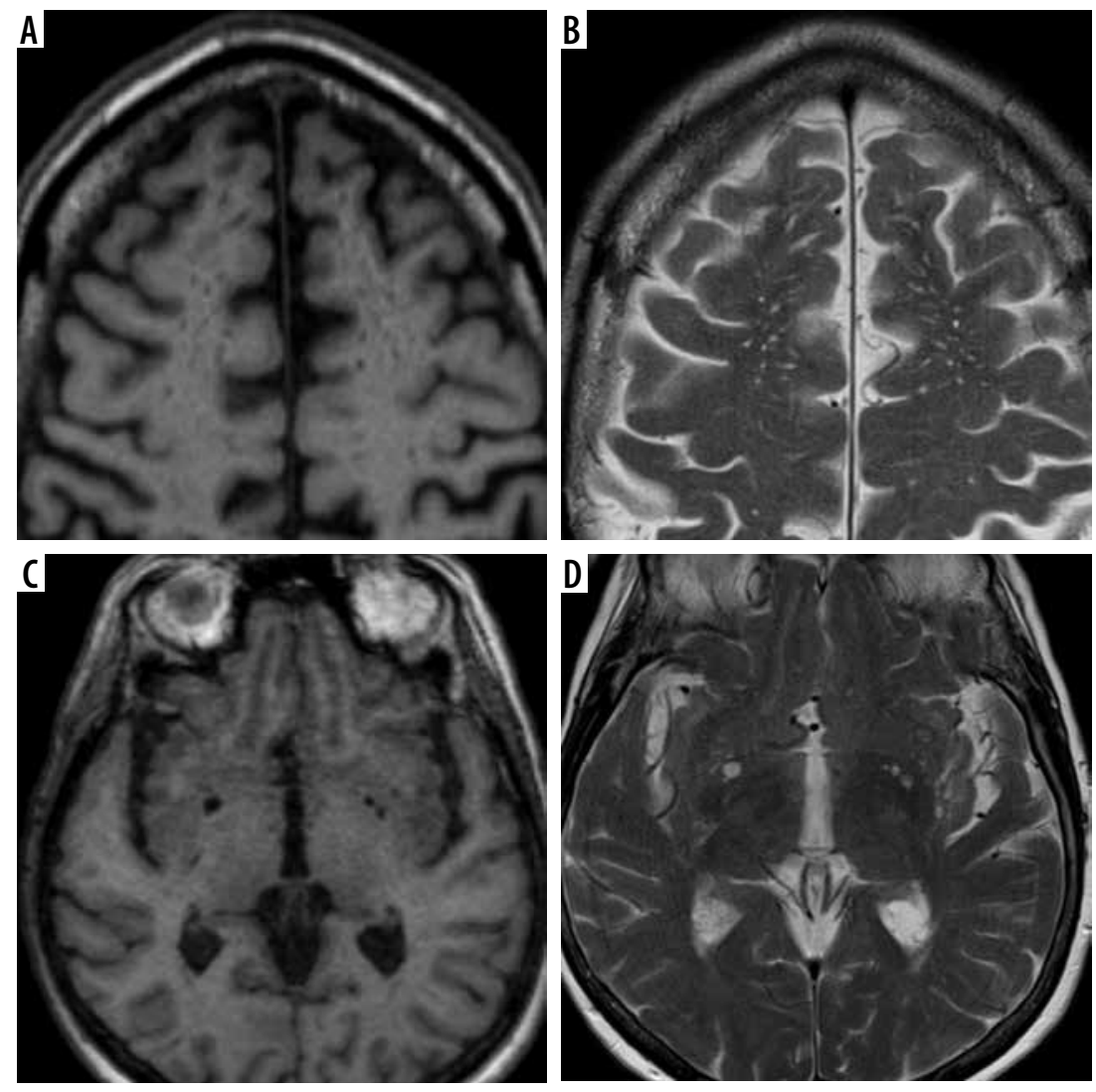

Figure 3. Enlarged perivascular spaces. A) Transverse T1-weighted image of enlarged perivascular spaces in the semi-oval centre. B) Transverse T2-weighted image of enlarged perivascular spaces in the semi-oval centre. C) Transverse T1-weighted image of enlarged perivascular spaces in the basal nuclei. D) Transverse T2-weighted image of enlarged perivascular spaces in the basal nuclei 

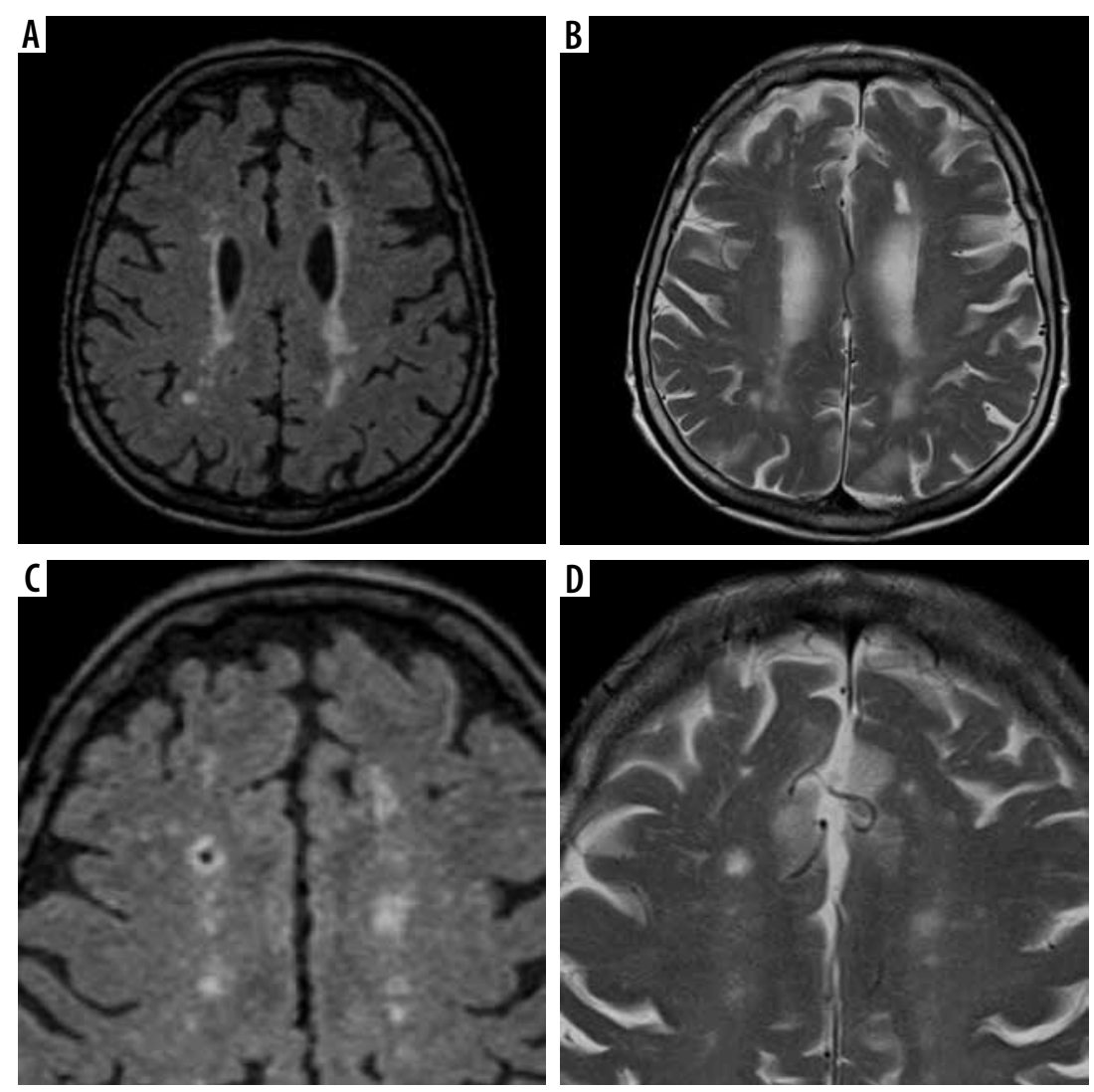

Figure 4. (erebral microbleeds. A) Transverse SWI image of microbleeds in the parietal lobes. B) Transverse SWI image of microbleeds in the parietal and occipital lobes. C) Transverse SWI image of microbleeds in the frontal, parietal, and occipital lobes and deep cerebral structures. D) Transverse SWI image of infratentorial microbleeds in the cerebellum and brainstem

the AS group showed higher variance in the number of the lacunar strokes. The results are presented in Table 2.

\section{Enlarged perivascular spaces}

Regardless of the analysed area, the prevalence of ePVS in the AS group and the control group was similar: in the semi-oval centre ( $97 \%$ vs. $98 \%, p=0.784)$, in the basal nu-

Table 1. Intensification of white matter hyperintensities in scale proposed by Fazekas et al. [10]

\begin{tabular}{|c|c|c|c|}
\hline & $\begin{array}{l}\text { Severe AS } \\
\text { group }\end{array}$ & $\begin{array}{l}\text { Control } \\
\text { group }\end{array}$ & $p$-value \\
\hline PVWM, $n$ (\%) & $27(79.41)$ & $28(46.00)$ & 0.027 \\
\hline Fazekas grade 0 & $7(20.59)$ & $22(44.00)$ & \multirow[t]{4}{*}{0.019} \\
\hline Fazekas grade 1 & $18(52.94)$ & $21(42.00)$ & \\
\hline Fazekas grade 2 & $7(20.59)$ & $7(14.00)$ & \\
\hline Fazekas grade 3 & $2(5.88)$ & $0(0.00)$ & \\
\hline DWM, $n(\%)$ & $26(76.47)$ & $33(66.00)$ & 0.303 \\
\hline Fazekas grade 0 & $8(23.53)$ & $17(34.00)$ & \multirow[t]{4}{*}{0.342} \\
\hline Fazekas grade 1 & $20(58.82)$ & $27(54.00)$ & \\
\hline Fazekas grade 2 & $4(11.77)$ & $6(12.00)$ & \\
\hline Fazekas grade 3 & $2(5.88)$ & $0(0.00)$ & \\
\hline
\end{tabular}

clei $(97 \%$ vs. $100 \%, p=0.360)$, and in the mesencephalon $(50 \%$ vs. $44 \%, p=0.591)$. Also, the density of the lesions determined using the scale proposed by Maclullich et al. [11] did not differ significantly between the study groups. The results are presented in Table 3.

\section{Cerebral microbleeds}

Overall, CMBs were found more often in patients with severe AS than in healthy controls. However, statistical analysis demonstrated that the study groups did not differ significantly in terms of the prevalence of CMBs in any of the analysed areas: in the deep cerebral structures (15\% vs. $14 \%, p=0.928)$, in the cerebral lobes $(20 \%$ vs. $12 \%$, $p=0.293)$, and in the infratentorial structures ( $14 \%$ vs. $8 \%$, $p=0.339$ ). Also, distributions of CMB density expressed on the MARS scale were similar in both study groups. The results are presented in Table 4.

Table 2. Prevalence and number of lacunar strokes

\begin{tabular}{|l|c|c|c|}
\hline & $\begin{array}{c}\text { Severe AS } \\
\text { group }\end{array}$ & $\begin{array}{c}\text { Control } \\
\text { group }\end{array}$ & $p$-value \\
\hline $\begin{array}{l}\text { Prevalence of lacunar strokes, } \\
n(\%)\end{array}$ & $12(35.29)$ & $8(16.00)$ & 0.042 \\
\hline $\begin{array}{l}\text { Number of lacunar strokes, } \\
\text { mean (SD) }\end{array}$ & $0.91(1.96)$ & $0.34(1.06)$ & 0.035 \\
\hline
\end{tabular}


Table 3. Intensification of enlarged perivascular spaces in scale proposed by Maclullich et al. [11]

\begin{tabular}{|c|c|c|c|}
\hline & $\begin{array}{l}\text { Severe AS } \\
\text { group }\end{array}$ & $\begin{array}{l}\text { Control } \\
\text { group }\end{array}$ & $p$-value \\
\hline ePVS, semioval centre, $n(\%)$ & $33(97.06)$ & $49(98.00)$ & 0.784 \\
\hline Maclullich grade 0 & $1(2.94)$ & $1(2.00)$ & \multirow[t]{5}{*}{0.844} \\
\hline Maclullich grade 1 & $8(23.53)$ & $13(26.00)$ & \\
\hline Maclullich grade 2 & $19(55.88)$ & $28(56.00)$ & \\
\hline Maclullich grade 3 & $5(14.71)$ & $7(14.00)$ & \\
\hline Maclullich grade 4 & $1(2.94)$ & $1(2.00)$ & \\
\hline ePVS, basal nuclei, $n(\%)$ & $33(97.06)$ & $50(100.00)$ & 0.360 \\
\hline Maclullich grade 0 & $1(2.94)$ & $0(0.00)$ & \multirow[t]{5}{*}{0.944} \\
\hline Maclullich grade 1 & $19(55.88)$ & $30(60.00)$ & \\
\hline Maclullich grade 2 & $8(23.53)$ & $12(24.00)$ & \\
\hline Maclullich grade 3 & $4(11.77)$ & $8(16.00)$ & \\
\hline Maclullich grade 4 & $2(5.88)$ & $0(0.00)$ & \\
\hline ePVS, mesencephalon, $n(\%)$ & $17(50.00)$ & $22(44.00)$ & 0.591 \\
\hline Maclullich grade 0 & $17(50.00)$ & $28(56.00)$ & \multirow[t]{5}{*}{0.515} \\
\hline Maclullich grade 1 & $16(47.06)$ & $22(44.00)$ & \\
\hline Maclullich grade 2 & $1(2.94)$ & $0(0.00)$ & \\
\hline Maclullich grade 3 & $0(0.00)$ & $0(0.00)$ & \\
\hline Maclullich grade 4 & $0(0.00)$ & $0(0.00)$ & \\
\hline
\end{tabular}

\section{Discussion}

The main findings in this study are that there is significantly increased prevalence of individual indices of CSVD in patients with severe AS. PVWM hyperintensities were found significantly more often $(p=0.027)$ in patients with severe AS than in the controls $(80 \%$ vs. $46 \%)$. Also, the density of the PVWM hyperintensities expressed on the Fazekas scale was significantly higher $(p=0.019)$. Lacunar strokes were found in 35\% of patients with severe AS and $16 \%$ of the healthy volunteers $(p=0.042)$. The average number of lacunar strokes per person was approximately 3 -fold higher in the AS group than in the controls (0.9 vs. 0.3, $p=0.035$ ). The analysis of the distribution and density of CMBs showed that, albeit insignificantly, our patients with AS differed from healthy volunteers in terms of the prevalence of these lesions in the cerebral lobes ( $20 \%$ vs. $12 \%, p=0.293)$ and infratentorial structures ( $14 \%$ vs. $8 \%$, $p=0.339$ ).

\section{White matter hyperintensities}

Published evidence shows that PVWM hyperintensities are over 4 times more common in patients with the diseases of large vessels, i.e. with TIA or stroke $(\mathrm{OR}=4.2)$ [13]. De Leeuw et al. reported a substantial density of these lesions in patients who presented with advanced athero-
Table 4. Intensification of cerebral microbleeds in Microbleed Anatomical Rating Scale proposed by Gregoire et al. [12]

\begin{tabular}{|l|c|c|c|} 
& $\begin{array}{c}\text { Severe AS } \\
\text { group }\end{array}$ & $\begin{array}{c}\text { Control } \\
\text { group }\end{array}$ & $p$-value \\
\cline { 1 - 3 } Prevalence of deep CMBs, $n$ (\%) & $5(14.71)$ & $7(14.00)$ & 0.928 \\
\hline Prevalence of lobar CMBs, $n$ (\%) & $7(20.59)$ & $6(12.00)$ & 0.293 \\
\hline Prevalence of infratentorial CMBs, $n$ (\%) & $5(14.71)$ & $4(8.00)$ & 0.339 \\
\hline MARS grade 0 & $22(64.71)$ & $37(74.00)$ & 0.427 \\
\hline MARS grade 1 & $10(29.41)$ & $9(18.00)$ & \\
\hline MARS grade 2 & $0(0.00)$ & $2(4.00)$ & \\
\cline { 1 - 3 } MARS grade 3 & $2(5.88)$ & $2(4.00)$ & \\
\hline
\end{tabular}

sclerosis of thoracic aorta at middle age. In such patients, the relative risk (RR) of white matter hyperintensities at an older age was estimated at 2.4. Interestingly, however, aortic atherosclerosis seemed not to predispose to the development of hyperintensities in the DWM $(\mathrm{RR}=$ 1.1). Also, atherosclerosis of thoracic aorta that developed at the age of more than 65 years was not associated with more frequent presence of PVWM hyperintensities $(\mathrm{RR}=1.0)[9]$. Another study conducted by the same group showed a correlation between the density of PVWM hyperintensities and the number of atherosclerotic plaques in the internal carotid artery $(p=0.19)$ or an increase of the intima-media thickness $(p=0.09)$. Importantly, also in that study, no significant correlations were found between the severity of the atherosclerotic lesions and the presence of DWM hyperintensities [14]. Moreover, Den Heijer et al. demonstrated that higher serum level of carotenoids, a factor protecting against carotid atherosclerosis, was associated with a lower number of hyperintensities in the PVWM, but not in the DWM [15].

On the other hand, DWM hyperintensities seem to be found more frequently in patients with arterial hypertension [16]. Thus, it has been postulated that PVWM and DWM hyperintensities are likely to have distinct pathomechanisms [17]. The fact that the PVWM is particularly prone to ischaemia stems from the anatomy of its vascular system; this area is supplied by long perforating arteries, branches of subarachnoid vessels that run from the cortex to the ventricular system (border zone and watershed areas) along myelin fibres. Because of its more stable vascular system, the DWM is less prone to hypoperfusion, whether systemic or local, and the development of DWM hyperintensities seems to be primarily associated with structural changes within the vessels, including those with smaller diameters [18-20].

Considering all the above, the significantly higher prevalence and density of PVWM hyperintensities in our patients with severe AS was probably associated with systemic hypoperfusion caused by chronic AS. This hypothesis seems to be supported also by the observations on the prevalence and density of DWM hyper- 
intensities, which did not differ between the study groups. Importantly, these are DWM hyperintensities, which are postulated to correlate strongly with CSVD and to be to a lesser degree linked to an impairment of systemic circulation.

\section{Lacunar strokes}

Lacunar strokes are a relatively common radiological finding; their prevalence increases with age, up to $8-28 \%$ in persons older than 60 years [21]. According to Regenhardt et al., the principal underlying mechanism of lacunar stroke is the occlusion of perforating arteries caused either by small atherosclerotic plaques or fibrosis and lipohyalinosis, i.e. fundamental processes involved in CSVD. However, the authors did not exclude other than CSVD aetiology of lacunar stroke [22]. Caplan found an association between the presence of atherosclerotic calcifications in the branches of large perforating arteries of the brain (branch atheromatous disease - BAD) and the number of lacunar strokes [23]. In another study, Tan et al. showed that the abnormalities of the large cerebral arteries (basilar artery, common carotid arteries, vertebral arteries, proximal and distal segments of anterior, middle, and posterior cerebral arteries) contributed to the stenosis of these vessels and resultant disorders of perfusion in up to $26 \%$ of 182 patients with lacunar stroke [24]. In turn, Del Brutto et al. demonstrated a relationship between the number of calcified atherosclerotic plaques in the carotid siphon and the occurrence of lacunar strokes. In a group of 236 participants with such lesions, lacunar strokes were more than 3 times more likely $(\mathrm{OR}=3.1)$ than in the controls [25]. Another study, conducted by Song et al., included 360 patients after ischaemic stroke. The authors analysed relationships between the characteristics of CSVD and the presence of atherosclerotic plaques in various segments of the thoracic aorta. Patients with lacunar strokes presented with atherosclerotic plaques in the thoracic aorta more than 4 times more often than the other participants $(\mathrm{OR}=4.24)[26]$.

In patients with severe AS and severe calcification of the aortic valve anulus, lacunar strokes might be a consequence of reduced cerebral perfusion or detachment of the calcified fragments and formation of arterio-arterial micro-emboli in small cerebral vessels. This calls into question the hypothesis that the significantly higher prevalence of lacunar strokes in patients with AS is primarily associated with CSVD.

\section{Enlarged perivascular spaces}

The prevalence and size of ePVS are known to increase substantially with age, although these lesions can also be found in younger persons. A link has been found between the more frequent occurrence of ePVS and arterial hypertension, a leading cause of CSVD. Moreover, the degree of PVS enlargement was shown to correlate with CSVD severity [27].

In a large Japanese study conducted by Yakushiji et al. in 1575 healthy adults, ePVS in the semi-oval centre were found in $99.6 \%$ of the participants and ePVS in the basal nuclei in $93.1 \%$; those observations are consistent with our findings [28]. In turn, Zhu et al. identified ePVS, whether in the white matter or basal nuclei, in all 1818 participants older than 65 years $[29,30]$, which also remains in agreement with the results of our present study. Slight discrepancies between the results published by the 2 groups mentioned above and our findings might derive from differences in the methodology of ePVS density assessment. Nevertheless, in our present study, grade 1 or 2 ePVS in the semi-oval centre was found in $89 \%$ and $92 \%$ of the patients and controls, respectively, whereas the prevalence rates of such lesions in the French and Japanese studies were slightly lower at $77 \%$ each. However, the prevalence of grade 1 and 2 ePVS in the basal nuclei was nearly the same at $89 \%$ and $84 \%$, respectively, in patients and controls participating in our study, versus $89 \%$ and $90 \%$ in the French and Japanese study, respectively.

Most studies analysing the prevalence of ePVS centred around the occurrence of these lesions in the semi-oval centre and basal nuclei. To the best of our knowledge, previously published studies, especially those involving large groups of patients, did not analyse the prevalence of ePVS in the mesencephalon; thus, our findings cannot be compared with the results of previous population-based studies. In the only published report on the occurrence of ePVS in the mesencephalon, Potter et al. analysed the severity of these lesions in 2 groups of subjects, 60 persons in total. Unfortunately, we cannot compare our findings with their results because they did not provide information about either the absolute numbers or the percentages of participants with ePVS in the mesencephalon [31].

Our findings suggest that the impairment of cerebral perfusion associated with severe AS does not have a profound effect on the prevalence and density of ePVS; this conclusion seems to be supported both by the results of our comparative analysis of the AS patients and controls and by the results of previous large population-based studies.

\section{Microbleeds}

The term 'microbleed' refers to small, round or oval areas formed as a result of blood extravasation into perivascular spaces and accumulation of erythrocyte degradation product, hemosiderin. The prevalence of CMBs in individuals older than 65 years is known to increase with age [32,33].

In a large Icelandic study of 1962 persons with a mean age of 76 years, Sveinbjornsdottir et al. estimated the prevalence of CMBs at $11.1 \%$. The CMBs were nearly twice as frequent in men than in women, with prevalence rates of 
$14.4 \%$ and $8.8 \%$, respectively [34]. In another large study involving 1062 Dutch subjects, Vernooij et al. found CMBs in $18 \%$ of persons aged $60-69$ years. The prevalence of CMBs in patients aged 70-79 years and 80-97 years was $31 \%$ and $38 \%$, respectively, which implied that the occurrence of these lesions might increase with age and that they are more common in persons older than 70 years [35]. Our findings are consistent with the results of the Dutch study; the slight disproportion in the prevalence of CMBs in patients with severe AS and healthy controls might be associated with the different mean age of the 2 groups: 76 and 73 years, respectively. Also, this observation seems to support the notion that the prevalence of CMBs increases with age and is markedly higher in persons older than 70 years. Another reason behind the between-group disproportion in the prevalence of CMBs in our patients and controls might be the different sex structure of the 2 groups. While the group of patients with severe AS consisted of 17 women and 17 men, the proportion of women among the controls was slightly higher (29 women vs. 21 men). As mentioned above, the prevalence of CMBs in men participating in the Icelandic study was nearly twice as high as in women; this might explain a slightly lower occurrence of CMBs in our control group.

The most common aetiologies of CMBs include cavernous cerebral malformations, diffuse axonal injury, as well as cerebral angiopathies underlying CSVD, i.e. amyloid and hypertensive angiopathy. The location of CMBs may be suggestive of their aetiology; in the case of amyloid angiopathy the lesions are located primarily in the cerebral lobes, whereas in hypertensive angiopathy they are typically found in the deep structures [36]. A large body of evidence supports these observations; for example, some studies demonstrated that reduced levels of cholesterol and APOE\&4 or increased deposition of $\mathrm{A} \beta$ amyloid in the brain were associated with the presence of CMBs in the cerebral lobes $[37,38]$. Importantly, Gregg et al. observed a higher prevalence of CMBs in the cerebral lobes of patients with chronic cerebral hypoperfusion, i.e. a condition that might also be present in persons with aortic valve stenosis, especially severe AS [39]. A higher prevalence of CMBs in the deep structures and infratentorial area of the brain has been previously linked to various pathologies of carotid arteries, such as atherosclerosis overall [40], the presence of calcified atherosclerotic plaques [42], and arterial stiffness [41]. In the case of all the pathologies mentioned above, the occurrence of CMBs in the deep structures and the infratentorial area was a consequence of arterial hypertension causing changes in carotid arteries and resultant hypertension in smaller perforating vessels.

To summarize, the results of all the studies mentioned above imply that higher prevalence of CMBs in cerebral hemispheres and deep structures of the brain is probably associated with different pathomechanisms, chronic cerebral hypoperfusion, and diseases of large arteries, respec- tively. However, none of these mechanisms fully explains the higher prevalence of CMBs in patients with chronic AS. If the increased prevalence of microbleeds in the hemispheres was a consequence of systemic hypoperfusion, a higher number of CMBs typical of structural changes in arterial vessels would be observed both in the deep and infratentorial structures of the brain. However, although our patients with severe AS presented with infratentorial CMBs more often than the controls, no significant between-group difference was found in the prevalence of CMBs in the deep cerebral structures. Thus, it can be hypothesized that patients with severe AS are characterized by a higher prevalence of CMBs in the cerebral hemispheres and infratentorial structures but not in the deep structures of the brain. However, this hypothesis needs to be verified in a larger group of patients.

There are limitations to this study. First, the small number of patients limits the statistical results. We recommended further studies with larger numbers of patients. Second, examinations were done using a 1.5 Tesla scanner, but further studies are recommended to be performed using a 3 Tesla scanner to improve the results. Third, in this study cerebral atrophy as a feature of CSVD was not included due to technical problems related to the volumetry assessment. In further studies, the assessment of cerebral atrophy will improve to evaluate the CSVD spectrum in AS patients. Fourth, there was no neurological correlation. Further studies with correlation between the prevalence of neuroradiological indices of CSVD and the neurological condition of the participants would help to better characterise the pathogenesis of CSVD in patients with severe AS.

\section{Conclusions}

Neuroradiological indices of the vascular disease do not provide an unequivocal clue to the pathogenesis of CSVD in patients with severe AS. Nevertheless, most observations made in patients with severe AS, namely increased prevalence of PVWM hyperintensities, lacunar strokes and CMBs in the cerebral lobes and infratentorial structures, imply that CSVD is primarily a consequence of cerebral hypoperfusion caused by AS. Patients with severe AS do not seem to differ from healthy persons in terms of the prevalence of the most characteristic indices of CSVD, DWM hyperintensities, and CMBs, as well as with regards to the occurrence of ePVS, a presumed risk factor of cerebral small vessel disease.

\section{Conflicts of interest}

The authors report no conflict of interest. 


\section{References}

1. GUS. Trwanie życia w 2018 r. [Life expectancy in 2018] stat.gov.pl. https://stat.gov.pl/obszary-tematyczne/ludnosc/trwanie-zycia/trwanie-zycia-w-2018-r-,2,13.html. Published: 2019.

2. Virani SS, Alonso A, Benjamin EJ, et al. Heart disease and stroke statistics - 2020 update: a report from the American Heart Association. Circulation 2020; 141: e139-e596.

3. Baumgartner H, Hung J, Bermejo J, et al. Recommendations on the echocardiographic assessment of aortic valve stenosis: a focused update from the European Association of Cardiovascular Imaging and the American Society of Echocardiography. J Am Soc Echocardiogr 2017; 30: 372-392.

4. Carabello BA, Paulus WJ. Aortic stenosis. Lancet 2009; 373: 956-966.

5. Pantoni L. Cerebral small vessel disease: from pathogenesis and clinical characteristics to therapeutic challenges. Lancet Neurol 2010; 9: 689-701.

6. Wardlaw JM, Smith EE, Biessels GJ, et al. Neuroimaging standards for research into small vessel disease and its contribution to ageing and neurodegeneration. Lancet Neurol 2013; 12: 822-838.

7. Cannistraro RJ, Badi M, Eidelman BH, et al. CNS small vessel disease: a clinical review. Neurology 2019; 92: 1146-1156.

8. de Leeuw FE, De Groot JC, Oudkerk M, et al. Aortic atherosclerosis at middle age predicts cerebral white matter lesions in the elderly. Stroke 2000; 31: 425-429.

9. Lazarus R, Prettyman R, Cherryman G. White matter lesions on magnetic resonance imaging and their relationship with vascular risk factors in memory clinic attenders. Int J Geriatr Psychiatry 2005; 20 : 274-279.

10. Fazekas F, Chawluk JB, Alavi A, et al. MR signal abnormalities at $1.5 \mathrm{~T}$ in Alzheimer's dementia and normal aging. AJR Am J Roentgenol 1987; 149: 351-356.

11. Maclullich AM, Wardlaw JM, Ferguson KJ, et al. Enlarged perivascular spaces are associated with cognitive function in healthy elderly men. J Neurol Neurosurg Psychiatry 2004; 75: 1519-1523.

12. Gregoire SM, Chaudhary UJ, Brown MM, et al. The Microbleed Anatomical Rating Scale (MARS): reliability of a tool to map brain microbleeds. Neurology 2009; 73: 1759-1766.

13. Lazarus R, Prettyman R, Cherryman G. White matter lesions on magnetic resonance imaging and their relationship with vascular risk factors in memory clinic attenders. Int J Geriatr Psychiatry 2005; 20: 274-279.

14. de Leeuw FE, de Groot JC, Bots ML, et al. Carotid atherosclerosis and cerebral white matter lesions in a population based magnetic resonance imaging study. J Neurol 2000; 247: 291-296.

15. den Heijer T, Launer LJ, de Groot JC, et al. Serum carotenoids and cerebral white matter lesions: the Rotterdam scan study. J Am Geriatr Soc 2001; 49: 642-646.

16. Khan U, Porteous L, Hassan A, Markus HS. Risk factor profile of cerebral small vessel disease and its subtypes. J Neurol Neurosurg Psychiatry 2007; 78: 702-706.

17. Kim KW, MacFall JR, Payne ME. Classification of white matter lesions on magnetic resonance imaging in elderly persons. Biol Psychiatry 2008; 64: 273-280.
18. Menshawi K, Mohr JP, Gutierrez J. A functional perspective on the embryology and anatomy of the cerebral blood supply. J Stroke 2015; 17: 144-158.

19. Moody DM, Bell MA, Challa VR. Features of the cerebral vascular pattern that predict vulnerability to perfusion or oxygenation deficiency: an anatomic study. AJNR Am J Neuroradiol 1990; 11: 431-439.

20. Pantoni L, Garcia JH, Gutierrez JA. Cerebral white matter is highly vulnerable to ischemia. Stroke 1996; 27: 1641-1647.

21. Vermeer SE, Longstreth WT Jr, Koudstaal PJ. Silent brain infarcts: a systematic review. Lancet Neurol 2007; 6: 611-619.

22. Regenhardt RW, Das AS, Lo EH, Caplan LR. Advances in understanding the pathophysiology of lacunar stroke: a review. JAMA Neurol 2018; 75: 1273-1281.

23. Caplan LR. Lacunar infarction and small vessel disease: pathology and pathophysiology. J Stroke 2015; 17: 2-6.

24. Tan MY, Singhal S, Ma H, et al. Examining subcortical infarcts in the era of acute multimodality CT imaging. Front Neurol 2016; 7: 220.

25. Del Brutto OH, Mera RM, Gillman J, et al. Calcifications in the carotid siphon correlate with silent cerebral small vessel disease in communitydwelling older adults: a population-based study in rural Ecuador. Geriatr Gerontol Int 2016; 16: 1063-1067.

26. Song TJ, Kim YD, Yoo J, et al. Association between aortic atheroma and cerebral small vessel disease in patients with ischemic stroke. J Stroke 2016; 18: 312-320.

27. Rouhl RP, van Oostenbrugge RJ, Knottnerus IL, et al. Virchow-Robin spaces relate to cerebral small vessel disease severity. J Neurol 2008; 255: 692-696.

28. Yakushiji Y, Charidimou A, Hara M, et al. Topography and associations of perivascular spaces in healthy adults: the Kashima scan study. Neurology 2014; 83: 2116-2123.

29. Zhu YC, Dufouil C, Mazoyer B, et al. Frequency and location of dilated Virchow-Robin spaces in elderly people: a population-based 3D MR imaging study. AJNR Am J Neuroradiol 2011; 32: 709-713.

30. Zhu YC, Tzourio C, Soumaré A, et al. Severity of dilated VirchowRobin spaces is associated with age, blood pressure, and MRI markers of small vessel disease: a population-based study. Stroke 2010; 41: 2483-2490.

31. Potter GM, Chappell FM, Morris Z, Wardlaw JM. Cerebral perivascular spaces visible on magnetic resonance imaging: development of a qualitative rating scale and its observer reliability. Cerebrovasc Dis 2015; 39: 224-231.

32. Janaway BM, Simpson JE, Hoggard N, et al. Brain haemosiderin in older people: pathological evidence for an ischaemic origin of magnetic resonance imaging (MRI) microbleeds. Neuropathol Appl Neurobiol 2014; 40: 258-269.

33. Sharma R, Dearaugo S, Infeld B, et al. Cerebral amyloid angiopathy: review of clinico-radiological features and mimics. J Med Imaging Radiat Oncol 2018; DOI: 10.1111/1754-9485.12726 [Online ahead of print].

34. Sveinbjornsdottir S, Sigurdsson S, Aspelund T, et al. Cerebral microbleeds in the population based AGES-Reykjavik study: prevalence and location. J Neurol Neurosurg Psychiatry 2008; 79: 1002-1006. 
35. Vernooij MW, van der Lugt A, Ikram MA, et al. Prevalence and risk factors of cerebral microbleeds: the Rotterdam Scan Study. Neuro$\operatorname{logy} 2008$; 70: 1208-1214.

36. Greenberg SM, Vernooij MW, Cordonnier C, et al. Cerebral microbleeds: a guide to detection and interpretation. Lancet Neurol 2009; 8: 165-174.

37. Romero JR, Preis SR, Beiser A, et al. Risk factors, stroke prevention treatments, and prevalence of cerebral microbleeds in the Framingham Heart Study. Stroke 2014; 45: 1492-1494.

38. Yates PA, Desmond PM, Phal PM, et al. Incidence of cerebral microbleeds in preclinical Alzheimer disease. Neurology 2014; 82: 1266-1273.
39. Gregg NM, Kim AE, Gurol ME, et al. Incidental cerebral microbleeds and cerebral blood flow in elderly individuals. JAMA Neurol 2015; 72: 1021-1028.

40. Romero JR, Preis SR, Beiser A, et al. Carotid Atherosclerosis and Cerebral Microbleeds: The Framingham Heart Study. J Am Heart Assoc 2016; 5: e002377.

41. Ding J, Mitchell GF, Bots ML, et al. Carotid arterial stiffness and risk of incident cerebral microbleeds in older people: the Age, Gene/ Environment Susceptibility (AGES)-Reykjavik study. Arterioscler Thromb Vasc Biol 2015; 35: 1889-1895.

42. Chung PW, Park KY, Kim JM, et al. Carotid artery calcification is associated with deep cerebral microbleeds. Eur Neurol 2014; 72: 60-63. 